\title{
Salvianolic acid B improves bone marrow-derived mesenchymal stem cell differentiation into alveolar epithelial cells type I via Wnt signaling
}

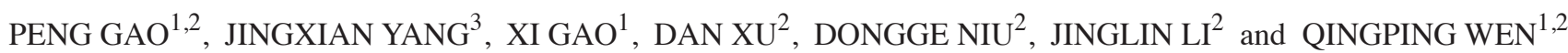 \\ ${ }^{1}$ Department of Anesthesiology, Dalian Medical University, Dalian, Liaoning 116044; \\ ${ }^{2}$ Department of Anesthesiology, First Affiliated Hospital of Dalian Medical University, Dalian, Liaoning 116011; \\ ${ }^{3}$ Department of Pharmacy, Liaoning University of Traditional Chinese Medicine, Dalian, Liaoning 116600, P.R. China
}

Received June 3, 2014; Accepted March 4, 2015

DOI: $10.3892 / \mathrm{mmr} .2015 .3632$

\begin{abstract}
Acute lung injury (ALI) is among the most common causes of mortality in intensive care units. Previous studies have suggested that bone marrow-derived mesenchymal stem cells (BMSCs) may attenuate pulmonary edema. In addition, alveolar epithelial cells type I (ATI) are involved in reducing the alveolar edema in response to ALI. However, the mechanism involved in improving the efficiency of differentiation of MSCs into ATI remains to be elucidated. In the present study, the effect of salvianolic acid B (Sal B) on the differentiation of BMSCs into ATI and the activities of the Wnt signaling pathways were investigated. The BMSCs were supplemented with conditioned medium (CM). The groups were as follows: i) CM group: BMSCs were supplemented with $\mathrm{CM}$; ii) lithium chloride ( $\mathrm{LiCl})$ group: BMSCs were supplemented with $\mathrm{CM}$ and $5 \mathrm{mM} \mathrm{LiCl}$; iii) Sal B group: BMSCs were supplemented with $\mathrm{CM}$ and $10 \mathrm{mM}$ Sal B. The samples were collected and assessed on days 7 and 14. It was revealed that aquaporin (AQP)-5 and T1 $\alpha$ were expressed in BMSCs, and induction with $\mathrm{LiCl}$ or Sal B increased the expression of AQP-5 and T1 $\alpha$. Furthermore, the Wnt-1 and Wnt-3a signaling pathways were activated during the differentiation of BMSCs into ATI. In conclusion, it was suggested that the promotive effects of Sal B on the differentiation of BMSCs into ATI occurred through the activation of Wnt signaling pathways.
\end{abstract}

\section{Introduction}

Acute lung injury (ALI) is a common complication following sepsis. Despite advances in supportive care and

Correspondence to: Professor Qingping Wen, Department of Anesthesiology, First Affiliated Hospital of Dalian Medical University, 222 Zhongshan Road, Dalian, Liaoning 116011, P.R. China E-mail: dmuwqp@163.com

Key words: salvianolic acid B, Wnt signaling pathway, acute lung injury, bone marrow-derived mesenchymal stem cells the pharmacological treatment of the condition, the mortality rates from ALI remain high (1). Previous studies have demonstrated that mesenchymal stem cells (MSCs) have therapeutic effects in the treatment of ALI, by reducing surface tension, thus preventing the alveoli from collapsing (2). MSCs can be engrafted in injured lung tissue and induced to differentiate into alveolar epithelial cells type II (ATII), which are vital in the maintenance of respiratory function through a reduction in surface tension, preventing collapse of the alveoli (3). In addition, it has also been revealed that MSCs may differentiate into alveolar epithelial cells type I (ATI), which are also associated with a reduction of pulmonary edema (4). However, the methods and mechanisms of improvement in the differentiation efficiency of MSCs into ATI remain to be elucidated.

Danshen [Salvia (S.) miltiorrhiza], a well-established Chinese Herbal Medicine, has been widely used in medicinal preparations for the treatment of ALI. Salvianolic acid B (Sal B) is one of the major constituents of the water-soluble extracts of S. miltiorrhiza Bunge, which has been reported to promote the differentiation of stem cells into cells of other lineages (5-7). It was thus hypothesized that Sal B possesses a promoting effect on the differentiation of stem cells into alveolar epithelial cells.

It has been reported that certain signaling pathways contribute to the induction of differentiation of MSCs into alveolar epithelial cells; therefore, it is important to examine the potential mechanisms in vitro. The Wnt signaling pathways have various roles in the differentiation of MSCs, dependent upon the specific ligands and receptors involved, as well as the target cells and the developmental stages (2-4). A previous study also demonstrated that the Wnt/ $\beta$-catenin signaling pathway is one of the fundamental pathways in cell proliferation, fate determination and the differentiation of MSCs, including the differentiation of MSCs into cells of an epithelial lineage (8). In addition, the Wnt/ $\beta$-catenin signaling pathway is a crucial regulator in tissue remodeling associated with lung diseases, including asthma, pulmonary fibrosis and chronic obstructive pulmonary disease $(9,10)$. Thus, it was hypothesized that the differentiation of stem cells into ATI occurs through the Wnt signaling pathways. 
CD29

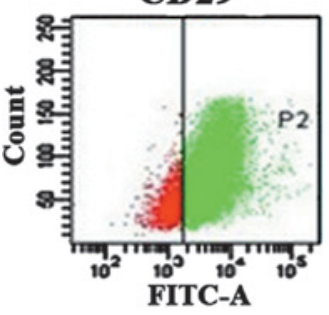

CD34

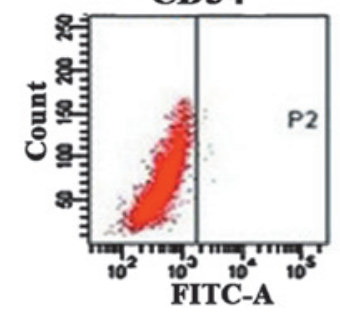

CD71

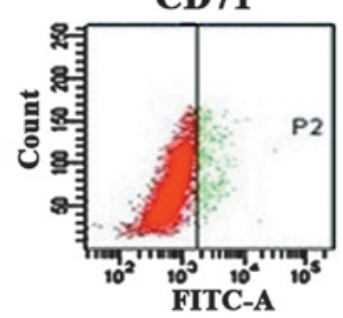

CD90

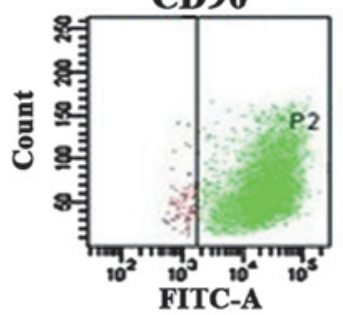

CD106

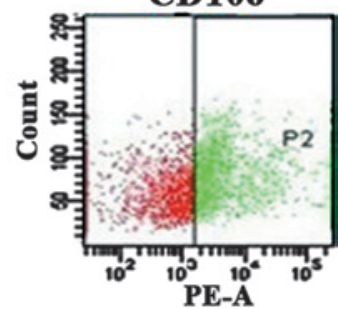

Figure 1. Flow cytometric analysis demonstrating the expression of BMSC surface markers. BMSCs were positive for expression of CD29 and CD90 (85.0 and $97.5 \%$, respectively). Furthermore, $47.2 \%$ of cells were CD106 positive, and the percentage of CD34- and CD71-expressing cells was low at 0.2 and $4.0 \%$, respectively. These values are averages from 3 repetitions. BMSC, bone marrow-derived mesenchymal stem cell; FITC, fluorescein isothiocyanate.

Considering the prospective effects of Sal B and Wnt signaling on the differentiation of bone marrow-derived mesenchymal stem cells (BMSCs), the present study was undertaken to investigate whether Sal B promoted the differentiation of BMSCs into ATI and whether this process was mediated by the activation of the Wnt signaling pathways.

\section{Materials and methods}

Ethical statement. All animal experimental procedures were approved by the Institutional Animal Care Committee of Dalian Medical University (Dalian, China). The present study was conducted according to institutional guidelines under an approved protocol. A total of 18 male Sprague Dawley (SD) rats (80-100 g body weight; 3-5 weeks; Specific Pathogen Free Laboratory Animal Center of Dalian Medical University, Dalian, China) were used in the present study.

Isolation and culture of BMSCs. BMSCs were isolated from the femur and tibia of two male SD rats as previously described (11). Briefly, the ends of the bones were cut, and the marrow was extruded with medium using a needle and syringe. The cell suspensions were plated on $25-\mathrm{cm}^{2}$ plastic flasks and supplemented with Dulbecco's modified Eagle's medium (DMEM) with $10 \%$ fetal bovine serum (FBS) and $1 \%$ glutamine (Cyagen Biosciences, Santa Clara, CA, USA). The cells were then incubated at $37^{\circ} \mathrm{C}$ with $5 \% \mathrm{CO}_{2}$. After incubation for $24 \mathrm{~h}$, the non-adherent cells were removed by replacing the medium. All experiments described in the present study were performed using cells from passages 5-8.

Flow cytometric analysis. Flow cytometric analysis of the expression of a panel of surface markers was performed using a BD Biosciences fluorescence-activated cell sorting machine (FACSAria2) using standard techniques (BD Biosciences, Mountain View, CA, USA). The BMSCs were harvested and washed with phosphate-buffered saline, then stained using antibodies against CD29, CD34, CD71, CD90 and CD106 (BD Biosciences).

BMSC differentiation assays. The lung homogenate was attained from the right lung of four SD rats $(80-100 \mathrm{~g})$, and was cultured without serum for $24 \mathrm{~h}$. Subsequently, the medium was concentrated using ultrafiltration units with a $3 \mathrm{kDa}$ molecular weight cutoff (Amicon, Billerica, MA, USA). The BMSCs were plated and supplemented with conditioned medium (CM), which contained a mixture of concentrated medium $(1 \mathrm{ml})$ and DMEM stem cell culture medium with $10 \%$ FBS and $1 \%$ glutamine $(1 \mathrm{ml})$ in equal volumes $(1: 1)$. The groups were as follows: i) CM group: BMSCs were supplemented with $\mathrm{CM}$; ii) lithium chloride ( $\mathrm{LiCl}$; Amresco LLC, Solon, OH, USA) group: BMSCs were supplemented with $\mathrm{CM}$ and $5 \mathrm{mM} \mathrm{LiCl}$; iii) Sal B group: BMSCs were supplemented with CM and $10 \mathrm{mM} \mathrm{Sal} \mathrm{B} \mathrm{(Sigma-Aldrich,} \mathrm{St.}$ Louis, MO, USA). All samples were collected and assessed on days 7 and 14.

Immunofluorescence staining. The cells were fixed in $4 \%$ paraformaldehyde (Solarbio, Beijing, China) and permeabilized with $0.1 \%$ Triton X-100 (Amresco LLC) for $15 \mathrm{~min}$ prior to incubation with the primary antibodies for $2 \mathrm{~h}$ at room temperature. The primary antibodies included rabbit polyclonal aquaporin (AQP)-5 (cat. no. sc-28628; 1:70 dilution; Santa Cruz Biotechnology, Inc., Santa Cruz, CA, USA), rabbit polyclonal T1 $\alpha$ (cat. no. bs-1048R; 1:70 dilution; Bioss, Shanghai, China), and fluorescein isothiocyanate-labeled donkey anti-rabbit secondary antibodies (cat. no. 711-095-152; 1:70 dilution; Jackson Immuno Research Inc., West Grove, PA, USA). The binding of DAPI (Sigma-Aldrich) to DNA was used to identify the nucleus. The immune complexes were observed using fluorescence microscopy (Olympus BX63; Olympus Corporation, Tokyo, Japan).

Western blot analysis. Total proteins were extracted from differentiated cells. The proteins were loaded onto a linear gradient polyacrylamide gel, with a polyacrylamide stacking gel, then electrophoretically transferred onto a polyvinylidene difluoride membrane following electrophoresis. The membranes were blocked via incubation with 5\% evaporated skimmed milk for blocking, followed by incubation with the primary antibodies at $4{ }^{\circ} \mathrm{C}$ overnight. The primary antibodies, including AQP-5 (1:200 dilution), T1 $\alpha$ (1:200 dilution), rabbit polyclonal Wnt-1 (cat. no. bs-1739R; 1:150 dilution; Bioss), rabbit polyclonal Wnt-3a (cat. no. bs-1700R; 1:150 dilution; Bioss) and mouse polyclonal $\beta$-actin (cat. no. sc-47778; 1:400 dilution; Santa Cruz Biotechnology, Inc.) were used. Following washing, the membranes were incubated with horseradish peroxidase-linked immunoglobulin $\mathrm{G}$ goat anti-rabbit or goat anti-mouse secondary antibodies (cat. nos. ZB-2305 or ZB-2301; 1:6,000 dilution; ZSGB-BIO, Beijing, China) for $2 \mathrm{~h}$ at room temperature. After standard enhanced chemiluminescence detection using gel-imaging with ChemiDoc XRS+ 

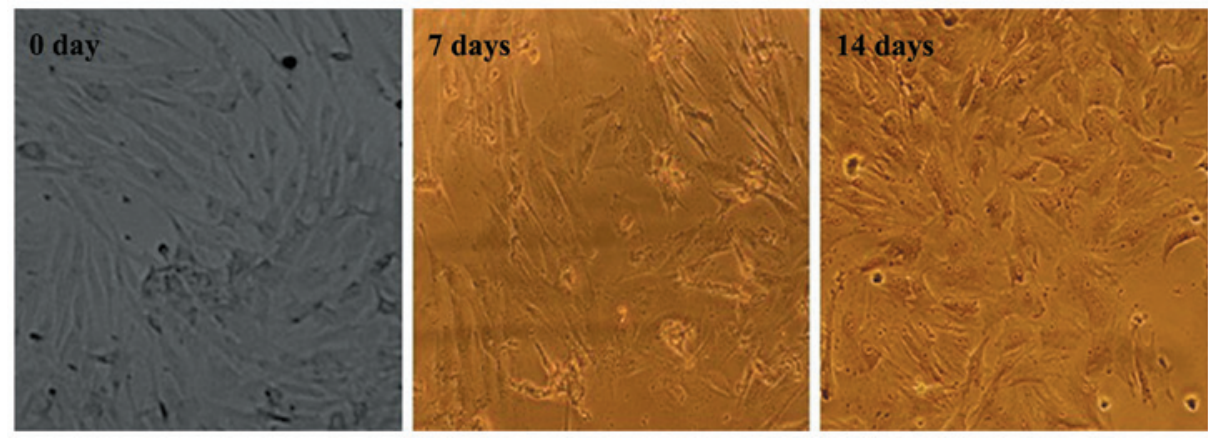

Figure 2. Morphological changes in BMSCs differentiated into alveolar epithelial cells. Under the inductive conditions, the BMSCs lost their characteristic fibroblast-like shape and gradually exhibited a polygonal morphology, typical of alveolar epithelial cells. On day 7, a few BMSCs exhibited an alveolar epithelial cell-like shape. On day 14, the majority of BMSCs exhibited morphology typical of alveolar epithelial cells. Images taken under differing light intensities, accounting for the variation in color. Magnification, $\mathrm{x} 40$. BMSCs, bone marrow-derived mesenchymal stem cells.
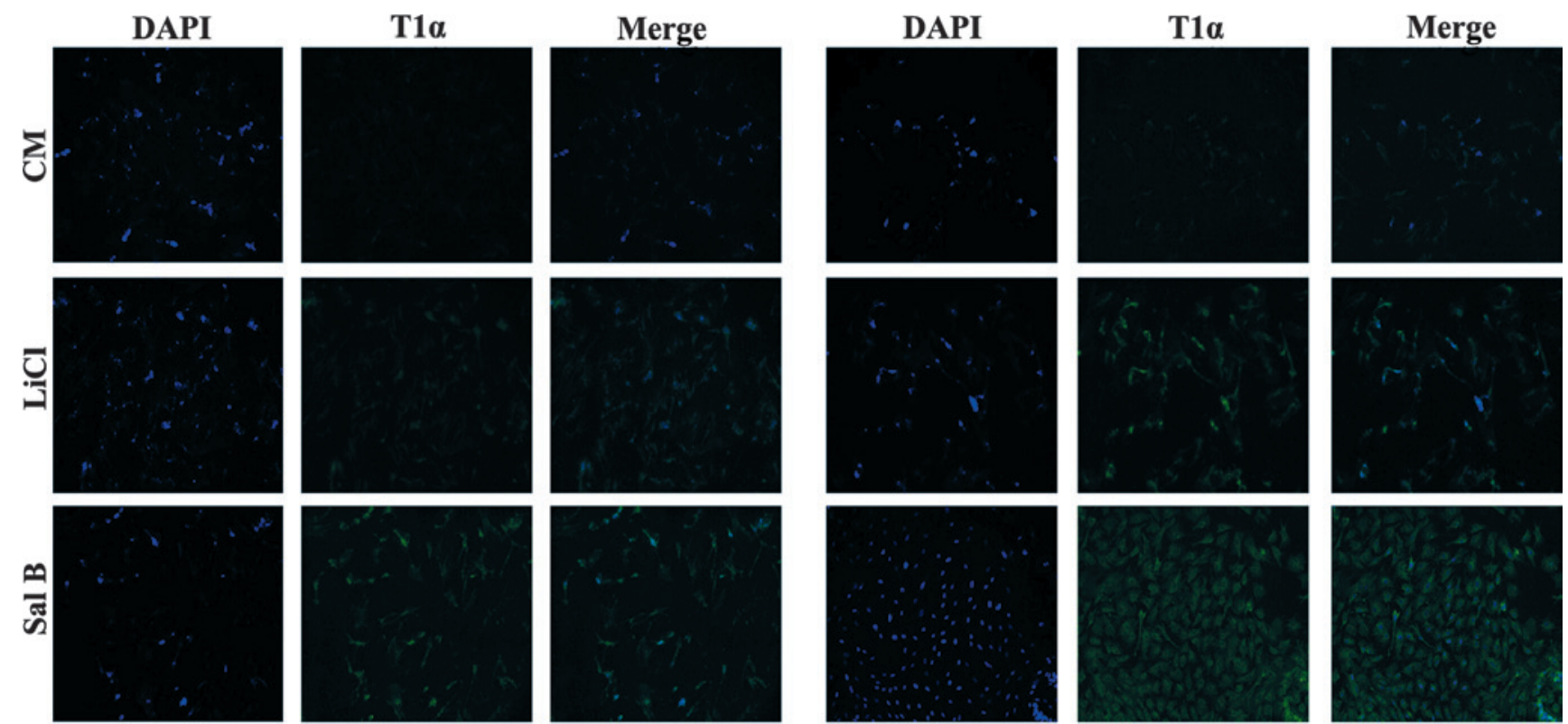

$7 \mathrm{~d}$

$14 \mathrm{~d}$
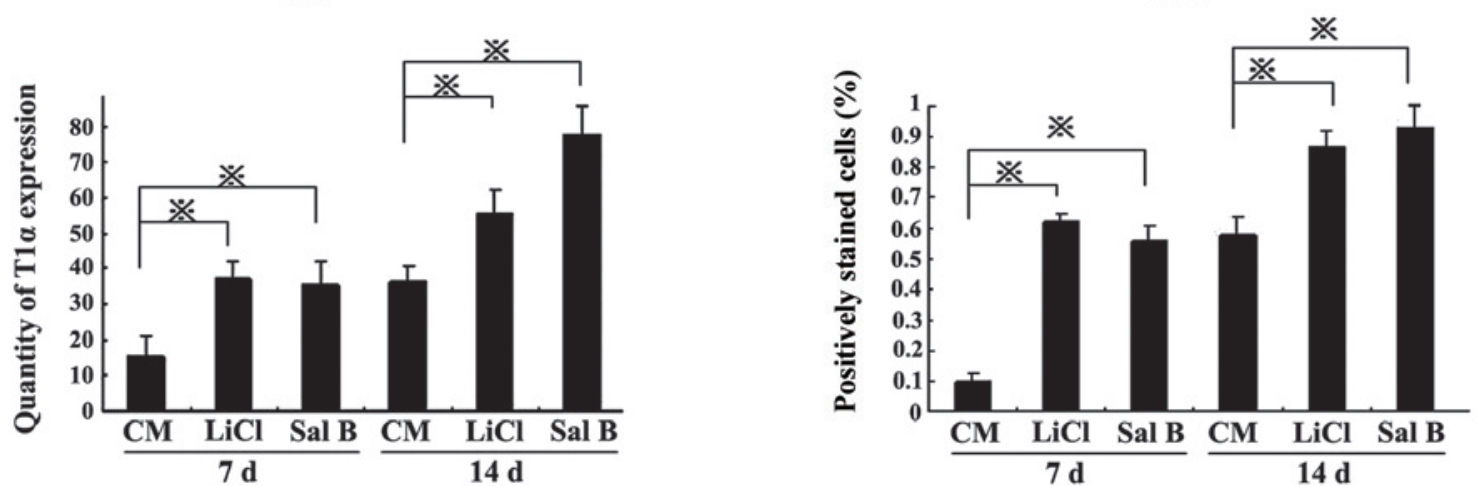

Figure 3. Bone marrow-derived mesenchymal stem cell differentiation increases expression of T1 $\alpha$. Under inductive conditions, the markers of the ATI lineage-specific marker, T1 $\alpha$, were examined. Induction with $\mathrm{LiCl}$ and Sal B increased $\mathrm{T} 1 \alpha$ expression. In the LiCl group and Sal B group, T1 $\alpha$ expression was significantly higher than that in the CM group at 7 and 14 days, respectively. Magnification, $\mathrm{x} 40$. ${ }^{*} \mathrm{P}<0.05$. Results are presented as the mean \pm standard deviation. CM, conditioned medium; ATI, alveolar epithelial cells type I; Sal B, salvianolic acid B.

(Bio-Rad Laboratories, Inc., Hercules, CA, USA) and Image Lab software, version 4.0 (Bio-Rad Laboratories, Inc.), the samples were subjected to image analysis using the ImageJ software, version 2.1 (National Institutes of Health, Bethesda, MD, USA).
Statistical analysis. Values are presented as the mean \pm standard error of the mean and were analyzed using SPSS 19.0 software (IBM, Armonk, NY, USA). The experimental data were analyzed using a one-way analysis of variance. $\mathrm{P}<0.05$ was considered to indicate a statistically significant difference. 

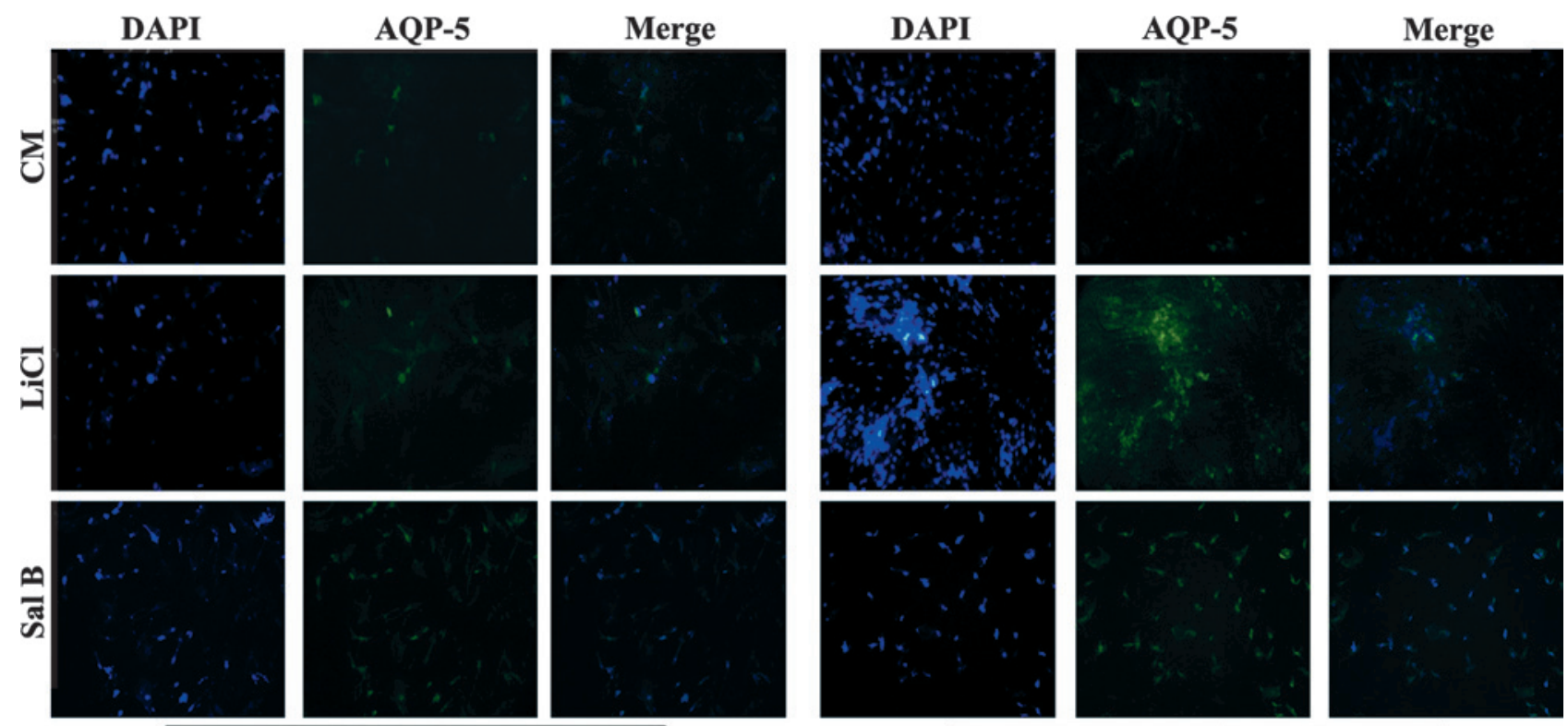

$7 \mathrm{~d}$

$14 \mathrm{~d}$
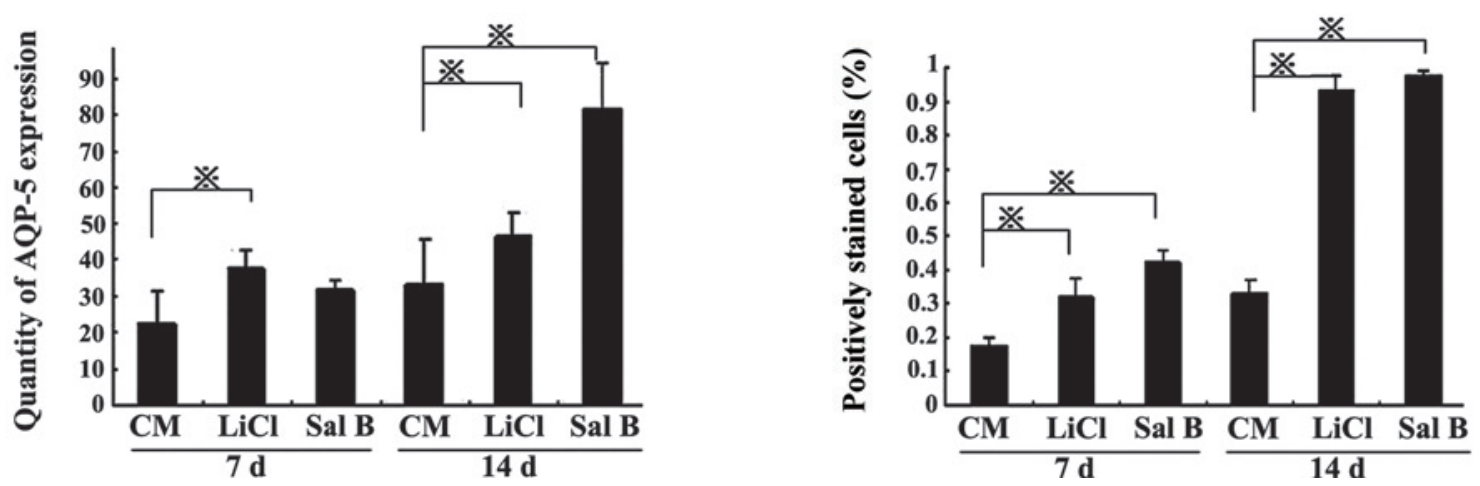

Figure 4. Bone marrow-derived mesenchymal stem cell differentiation increases expression of AQP-5. Under inductive conditions, the markers of the ATI lineage-specific marker, AQP-5, were examined. Induction with $\mathrm{LiCl}$ and $\mathrm{Sal} \mathrm{B}$ increased AQP-5 expression. In the LiCl group, the AQP-5 expression was significantly higher than that in the CM group at 7 and 14 days. In the Sal B group, the AQP-5 expression was significantly higher than that in the CM group on day 14. Magnification, $\mathrm{x} 40 .{ }^{*} \mathrm{P}<0.05$. Results are presented as the mean \pm standard deviation. $\mathrm{CM}$, conditioned medium; ATI, alveolar epithelial cells type I; Sal B, salvianolic acid B; AQP, aquaporin.

\section{Results}

Characteristics of BMSCs in culture. The majority of cells exhibited a spindle-like or fibroblast-like shape. Flow cytometric analysis demonstrated the expression of BMSC surface markers. BMSCs were positive for CD29 and CD90, (85.0 and $97.5 \%$, respectively). Expression of CD106 was present on $47.2 \%$ of BMSCs, while CD34 and CD71 expression was rare (0.18 and 4\%, respectively) (Fig. 1).

Morphological changes of BMSCs during differentiation into ATI. Under inductive conditions, the BMSCs lost their characteristic fibroblast-like shape and gradually began to exhibit a polygonal morphology, typical of ATI. On day 7, a few BMSCs exhibited an alveolar epithelial-like shape; however, by day 14, the majority of BMSCs exhibited the morphology indicative of differentiation into ATI (Fig. 2).

BMSC differentiation and expression of ATI markers. Under inductive conditions (stimulation with $\mathrm{LiCl}$ or $\mathrm{Sal} \mathrm{B}$ ), the expression of ATI lineage-specific markers, AQP-5 and T1 $\alpha$, was examined using immunofluorescence. BMSCs under control conditions expressed these proteins at relatively low levels on day 7; however, when BMSCs differentiated into ATI, AQP-5 and T1 $\alpha$ levels were increased by day 14 and following induction with $\mathrm{LiCl}$ or $\mathrm{Sal} \mathrm{B}$ compared with the CM group ( $\mathrm{P}<0.05$; Figs. 3 and 4$)$.

The western blot analysis revealed similar results to those of the immunofluorescence staining. In the Sal B group, the T1 $\alpha$ and AQP-5 protein levels were significantly increased compared with the $\mathrm{CM}$ group on days 7 and $14(\mathrm{P}<0.05)$. In the $\mathrm{LiCl}$ group, the T1 $\alpha$ and AQP-5 protein levels were significantly increased compared with those in the $\mathrm{CM}$ group on day 14 ( $\mathrm{P}<0.05$; Fig. 5).

Wnt pathway activation during the differentiation of BMSCs into ATI. Differentiation of BMSCs into ATI activated Wnt signaling, as indicated by western blot analysis of Wnt-1 and $-3 \mathrm{a}$. In the $\mathrm{LiCl}$ and Sal B groups, Wnt-1 levels were significantly higher that those in the CM group on day 7 

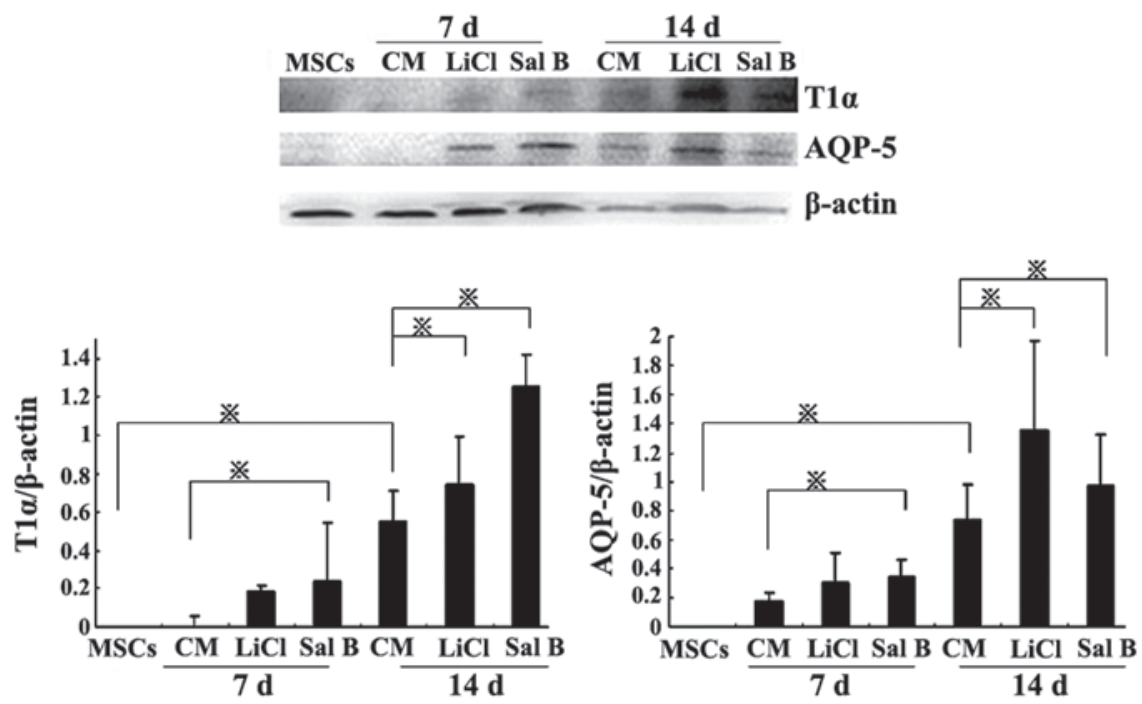

Figure 5. Differentiation of bone marrow-derived mesenchymal stem cells significantly increased the protein expression of T1 $\alpha$ and AQP-5 at 14 days. In the $\mathrm{Sal} \mathrm{B}$ and $\mathrm{LiCl}$ induction groups, T1 $\alpha$ and AQP-5 protein levels were significantly higher than those in the CM group at 7 and 14 days. Furthermore, following 14 days of induction, T1 $\alpha$ and AQP-5 protein levels were higher than those following 7 days. "P $<0.05$. Results are presented as the mean \pm standard deviation. CM, conditioned medium; Sal B, salvianolic acid B; AQP, aquaporin.

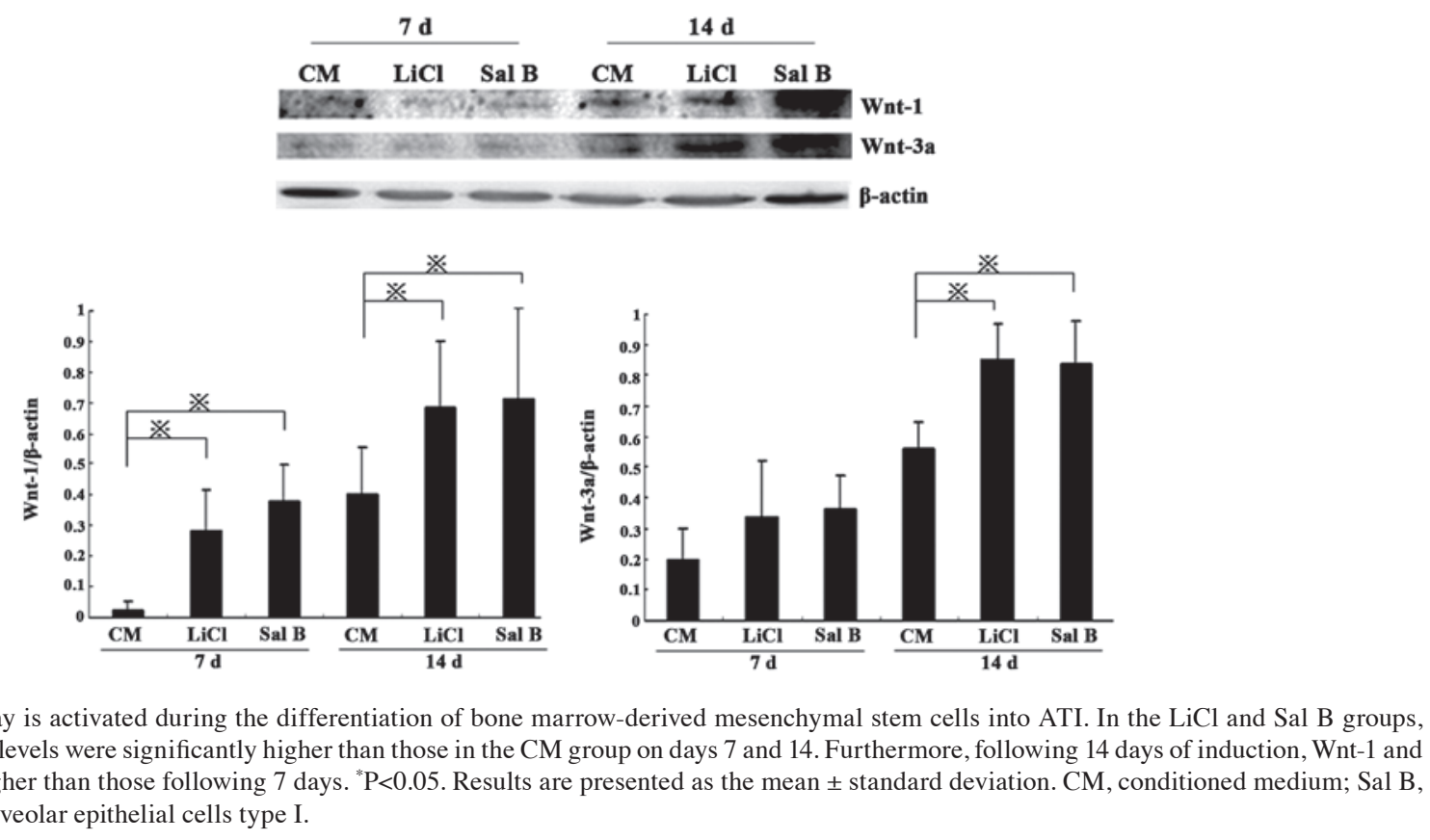

$(\mathrm{P}<0.05)$, and Wnt-1 and -3 a levels were further increased by day 14 (P<0.05; Fig. 6).

\section{Discussion}

Damage to the alveolar epithelium has been documented in $\sim 90 \%$ of patients with lung injury, according to data collected from lung biopsies (12). Lung tissue may be recovered with adequate repair and remodeling of the alveolar epithelia, but not in the case of severe injury and without the rapid activation of repair mechanisms. As stem cells are considered to differentiate into ATI as a replacement of injured alveolar epithelial cells, it was important to elucidate whether the BMSCs may be a suitable source of MSCs (3). BMSCs as a source of multipo- tent cells can be easily identified by detection of cell surface antigens; they are positive for CD29 and CD90, have low expression of CD106, and are negative for CD34 and CD71. The results of the present study confirmed findings from a previous study (11).

The supernatant of the lung tissue homogenate was used in the present study to investigate the mechanism of increasing the differentiation efficiency of stem cells into ATI by induction with Sal B. The results revealed that the cultured BMSCs had a polygonal morphology and expressed T1 $\alpha$ and AQP-5, which are specific markers for ATI. It was suggested that the differentiated cells serve as a precursor to the population of T1 $\alpha$-positive cultured cells in vivo (4). AQP-5 is an important mediator of the movement of water across the 
cell membranes of vascular endothelial cells and ATI via a transcellular pathway. In addition, in the case of pulmonary edema following injury, AQP-5 is expressed decreasingly in lung tissue (13). Therefore, it is expected that, by expressing AQP-5 and exhibiting the specific features of ATI, the stem cells (BMSCs) may differentiate into ATI and possibly be involved in the movement of water across the cell membranes to reduce alveolar edema in response to ALI.

In the present study, the effect of Sal B on the differentiation of BMSCs into ATI was examined. The natural product Sal B is an active component of S. miltiorrhiza, an herb widely used in Traditional Chinese Medicine. Accumulating evidence has suggested that Sal B has protective effects under various medical conditions. It has been reported that Sal B is able to significantly attenuate the decrease in viability of BMSCs subjected to damage through administration of potentially lethal $\mathrm{H}_{2} \mathrm{O}_{2}$, exhibiting time-dependent protective effects (14). The marked oxidative stress status in vivo also prevented the implanted stem cells from migrating and differentiating, eventually inducing apoptosis, resulting in an impaired effect of this promising therapy in the treatment of disease. The major pharmacological action of Sal B is to protect the pulmonary microcirculation from disturbance, inflammation and apoptosis $(15,16)$. In addition, Sal B increases the expression of AQP-5 in lung tissue in response to ALI (17). Sal B has been revealed to improve the differentiation capacity of stem cells into cells of other lineages, including neurons and cardiomyocytes $(6,7)$. In the present study, it was demonstrated that Sal B improves the differentiation efficiency of BMSCs into ATI.

It has been well-established that the Wnt signaling pathways are fundamental in cell proliferation, cell polarity and determination during embryonic development, and tissue homeostasis (18). This pathway may be activated when Wnt ligands, including Wnt-1, -2 , or $-3 \mathrm{a}$, are bound to the co-receptors of Frizzled and low-density lipoprotein receptor-related protein 5 or 6 . Furthermore, these binding events are able to lead to the inhibition of the Axin complex and glycogen synthase kinase (GSK) $3 \beta$, resulting in the dephosphorylation and stabilization of $\beta$-catenin in the cytoplasm, which subsequently translocates into the nucleus to regulate target gene expression (19). It is well-established that the accumulation of $\beta$-catenin is critical in inducing the Wnt signaling cascades (20). It was observed in the present study that Wnt-1 and -3a may be detected during the differentiation of BMSCs into ATI between 7 and 14 days. $\mathrm{LiCl}$ activates Wnt signaling by inhibiting GSK $3 \beta$ and consequently stabilizing free cytosolic $\beta$-catenin. Therefore, $\mathrm{LiCl}$ was used to investigate the regulation of the $\mathrm{Wnt} / \beta$-catenin signaling pathway in the differentiation of BMSCs into ATI. It was demonstrated that $\mathrm{LiCl}$ improved the differentiation efficiency of BMSCs into ATI, regulated by the Wnt signaling pathway. In addition, Sal B also markedly increased the differentiation efficiency of BMSCs through the activation of Wnt signaling pathways.

In the present study, it was suggested that Sal B possesses promotory effects on the differentiation of BMSCs into ATI, which is likely to be mediated by the activation of Wnt signaling.

\section{Acknowledgements}

The present study was supported by the National Nature Science Fund of China (grant no. 81273923).

\section{References}

1. Rubenfeld GD, Caldwell E, Peabody E, et al: Incidence and outcomes of acute lung injury. N Engl J Med 353: 1685-1693, 2005.

2. Ortiz LA, Gambelli F, McBride C, et al: Mesenchymal stem cell engraftment in lung is enhanced in response to bleomycin exposure and ameliorates its fibrotic effects. Proc Natl Acad Sci USA 100: 8407-8411, 2003.

3. Rojas M, Xu J, Woods CR, et al: Bone marrow derived mesenchymal stem cells in repair of the injured lung. Am J Respir Cell Mol Biol 33: 145-152, 2005.

4. Kotton DN, Ma BY, Cardoso WV, Sanderson EA, Summer RS, et al: Bone marrow-derived cells as progenitors of lung alveolar epithelium. Development 128: 5181-5188, 2001.

5. Zhuang P, Zhang Y, Cui G, Bian Y, Zhang M, Zhang J, Liu Y, Yang X, Isaiah AO, Lin Y, et al: Direct stimulation of adult neural stem/progenitor cells in vitro and neurogenesis in vivo by salvianolic acid B. PLoS One 7: e35636, 2012.

6. Guo G, Li B, Wang Y, Shan A, Shen W, Yuan L and Zhong S: Effects of salvianolic acid B on proliferation, neurite outgrowth and differentiation of neural stem cells derived from the cerebral cortex of embryonic mice. Sci China Life Sci 53: 653-662, 2010

7. Chan SS, Chen JH, Hwang SM, Wang IJ, Li HJ, Lee RT and Hsieh PC: Salvianolic acid B-vitamin C synergy in cardiac differentiation from embryonic stem cells. Biochem Biophys Res Commun 387: 723-728, 2009

8. Wang Y, Sun Z, Qiu X, Li Y, Qin J and Han X: Roles of Wnt/beta-catenin signaling in epithelial differentiation of mesenchymal stem cells. Biochem Biophys Res Commun 390: 1309-1314, 2009.

9. Lee H, Bae S, Choi BW and Yoon Y: WNT/ $\beta$-catenin pathway is modulated in asthma patients and LPS-stimulated RAW264.7 macrophage cell line. Immunopharmacol Immunotoxicol 34: 56-65, 2012.

10. Baarsma HA, Spanjer AI, Haitsma G, et al: Activation of WNT/ $\beta$-catenin signaling in pulmonary fibroblasts by TGF- $\beta_{1}$ is increased in chronic obstructive pulmonary disease. PLoS One 6: e25450, 2011.

11. Ionescu L, Byrne RN, van Haaften T, et al: Stem cell conditioned medium improves acute lung injury in mice: in vivo evidence for stem cell paracrine action. Am J Physiol Lung Cell Mol Physiol 303: L967-L977, 2012.

12. Parambil JG, Myers JL, Aubry MC and Ryu JH: Causes and prognosis of diffuse alveolar damage diagnosed on surgical lung biopsy. Chest 132: 50-57, 2007.

13. Gao. C, Li R, Huan J, et al: Caveolin-1 siRNA increases the pulmonary microvascular and alveolar epithelial permeability in rats. J Trauma 70: 210-219, 2011.

14. Lu B, Ye Z, Deng Y, Wu H and Feng J: MEK/ERK pathway mediates cytoprotection of salvianolic acid $\mathrm{B}$ against oxidative stress-induced apoptosis in rat bone marrow stem cells. Cell Biol Int 34: 1063-1068, 2010.

15. Lay IS, Hsieh CC, Chiu JH, Shiao MS, Lui WY and Wu CW: Salvianolic acid $\mathrm{B}$ enhances in vitro angiogenesis and improves skin flap survival in Sprague-Dawley rats. J Surg Res 115: 279-285, 2003.

16. Chen YL, Hu CS, Lin FY, et al: Salvianolic acid B attenuates cyclooxygenase-2 expression in vitro in LPS-treated human aortic smooth muscle cells and in vivo in the apolipoprotein-E-deficient mouse aorta. J Cell Biochem 98: 618-631, 2006.

17. Lin F, Liu YY, Xu B, et al: Salvianolic acid B protects from pulmonary microcirculation disturbance induced by lipopolysaccharide in rat. Shock 39: 317-325, 2013.

18. Logan CY and Nusse R: The Wnt signaling pathway in development and disease. Annu Rev Cell Dev Biol 20: 781-810, 2004.

19. Gordon MD and Nusse R: Wnt signaling: Multiple pathways, multiple receptors, and multiple transcription factors. J Biol Chem 281: 22429-22433, 2006.

20. MacDonald BT, Tamai K and He X: Wnt/beta-catenin signaling: Components, mechanisms and diseases. Dev Cell 17: 9-26, 2009. 